\title{
ANCORA SULLA VELOCITA' DI PROPAGAZIONE DELLE ONDE SUPERFICIALI PER TRAGITTI SUBATLANTICI. TENTATIVO DI PROSPEZIONE PROFONDA DEL BACINO ATLANTICO, MEDIANTE LE CURVE DI DISPERSIONE DELLE ONDE $L_{*}$
}

\author{
P. Caloi - L. Marcelli - G. Pannocchia
}

1. - Riassumiamo in questa prima parte alcune considerazioni sulle conclusioni ottenute in un nostro precedente lavoro $i-1$ ed aggiungiamo altri argomenti a favore delle conclusioni stesse.

Le velocità per le $L_{q}$ riportate per Yersey e per Tortosa sono sensibilmente minori di quelle osservate a Ottawa.

a) Non è consentito attribuire ai retroterra delle opposte sponde dell'Atlantico le stesse caratteristiche elastiche: in particolare, la vasta zona della Nuova Inghilterra e dell'Ontario, che si estende dietro Harvard ed interessa Ottawa, ha caratteristiche ben diverse da quelle della zona delle isole britanniche. Don Leet, J. H. Hogdson $\left({ }^{2}\right)$ ed altri hanno trovato per il primo velocità per le onde longitudinali dirette dell'ordine di $6,2 \mathrm{~km} / \mathrm{sec}$, laddove per le isole britanniche Jeffreys trova valori di $5,4 \mathrm{~km} / \mathrm{sec}$. Per le stazioni del retroterra può giocare un ruolo sensibile sulla velocità delle onde superficiali anche l'azione di rifrazione determinata dallo zoccolo continentale $\left({ }^{3}\right)$.

Pure prescindendo da ciò, per quanto concerne le stazioni di Jersey, Tortosa e Barcellona, dobbiamo osservare che, a motivo della limitata sensibilità degli apparecchi ivi funzionanti, la registrazione fu di lieve intensità e noi, per ovvia prudenza, abbiamo segnato l'inizio delle $L_{4}$ dove ci parve sicuro. Però, uno sguardo a dette registrazione prova che l'inizio delle onde stesse poteva essere anticipato di un minuto almeno. Ad og̣ni modo, a togliere ogni dubbio sulla forte velocità delle onde $L_{a}$ anche nel bacino orientale dell'Atlantico, oltre alla stazione di Lisbona, c'è la testimonianza ben più inoppugnabile di Kew (di cui, per una strana dimenticanza, abbiamo trascurato, nel lavoro precedente, la registrazione delle $L_{Q}$ ): qui le onde $L_{q}$ appaiono nettissime, inequivocabili e mostrano una velocità anche maggiore di quella registrata ad Ottawa, di $4,3 \mathrm{~km} / \mathrm{sec}$ almeno (fig. 1). 
b) Senza dublio, la sovrapposizione degli epicentri nell'arco mediterraneo è in parte fittizia, grande essendo il numero degli osservatori nelle zone limitrofe; però la densità degli epicentri risulta notevolissima anche in estese zone disabitate. A questo riguardo, la notevole densità degli epicentri che si osserva lungo tutta la soglia cen-

Kew Observatory $\cdot N-S \cdot \Delta t=-19,6-\Delta=5937 \mathrm{~km}$

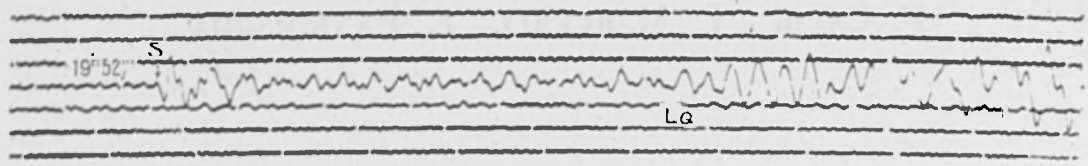

Fig. 1

trale atlantica, rispetto a quella debolissima relativa al tratto AzzorrePenisola Iberica è una prova assai chiara del difetto di saldatura tra le due zone sismiche: mediterranea e centro-atlantica.

c) A complemento di quanto è stato detto in proposito nella nota precedente, dobbiamo ricordare che la batimetria riprodotta nella nostra nota è quella ufficiale, dedotta dalla "Carte Générale Batliymétrique des Océans» del "Bureau Hydrograplique International ) di Monaco Principato, Ente internazionalmente autorizzato per tali ricerche ed espressamente attrezzato a questo scopo. La riproduzione si riferisce all'edizione più recente (1936, 3" ediz.). Qualsiasi altra fonte non puó essere clie parziale a questo riguardo e non può consentire quella fiducia che spetta invece all'Istituto di Monaco.

Ora, anche la batimetria ì in netto appoggio alle nostre conclusioni.

d) Come è noto, le velocità consentite dal mezzo solido sopportante l'Atlantico, erano ritenute avere valori intermedi fra quelli osservati per tragitti subpacifici e per tragitti continentali. Abbiamo provato, nel lavoro precedente, che in realtà le velocità delle onde sismiche per tragitti subatlantici coincidono praticamente con quelle che interessano il Pacifico.

Riteniamo che la cennata erronea conclusione sia stata frutto e di precedenti errate valutazioni e di malintesi. Una notevole influenza a favore di detta conclusione spetta indubbiamente ad un lavoro di Rohrbach del $1932\left({ }^{4}\right)$. In detto lavoro, Rohrbaclı considera, fra l'altro, le registrazioni ottenute ad Apia (Samoa) e a Gottinga, in occasione di un forte terremoto avvenuto sul bordo occidentale del Messico. Per quanto riguarda il tragitto delle onde superficiali, egli 
ritiene che quello verso Apia interessi il Pacifico (e ciò risponde effettivamente a verita), mentre considera come subatlantico il cammino delle stesse onde verso Gottinga. Dalle curve di dispersione, trova una notevole diversità: mentre onde superficiali di $100 \mathrm{~km}$ di lunghezza d'onda si propagano sotto il Pacifico con velocità dell'ordine di $4,0 \mathrm{~km} / \mathrm{sec}$, onde di uguale lunghezza d'onda non raggiungerebbero sotto l'Atlantico la velocità di $3,5 \mathrm{~km} / \mathrm{sec}$. Senonché, anche da un semplice esame della fig. 2 (12; nella nota di Rohrbach। appare

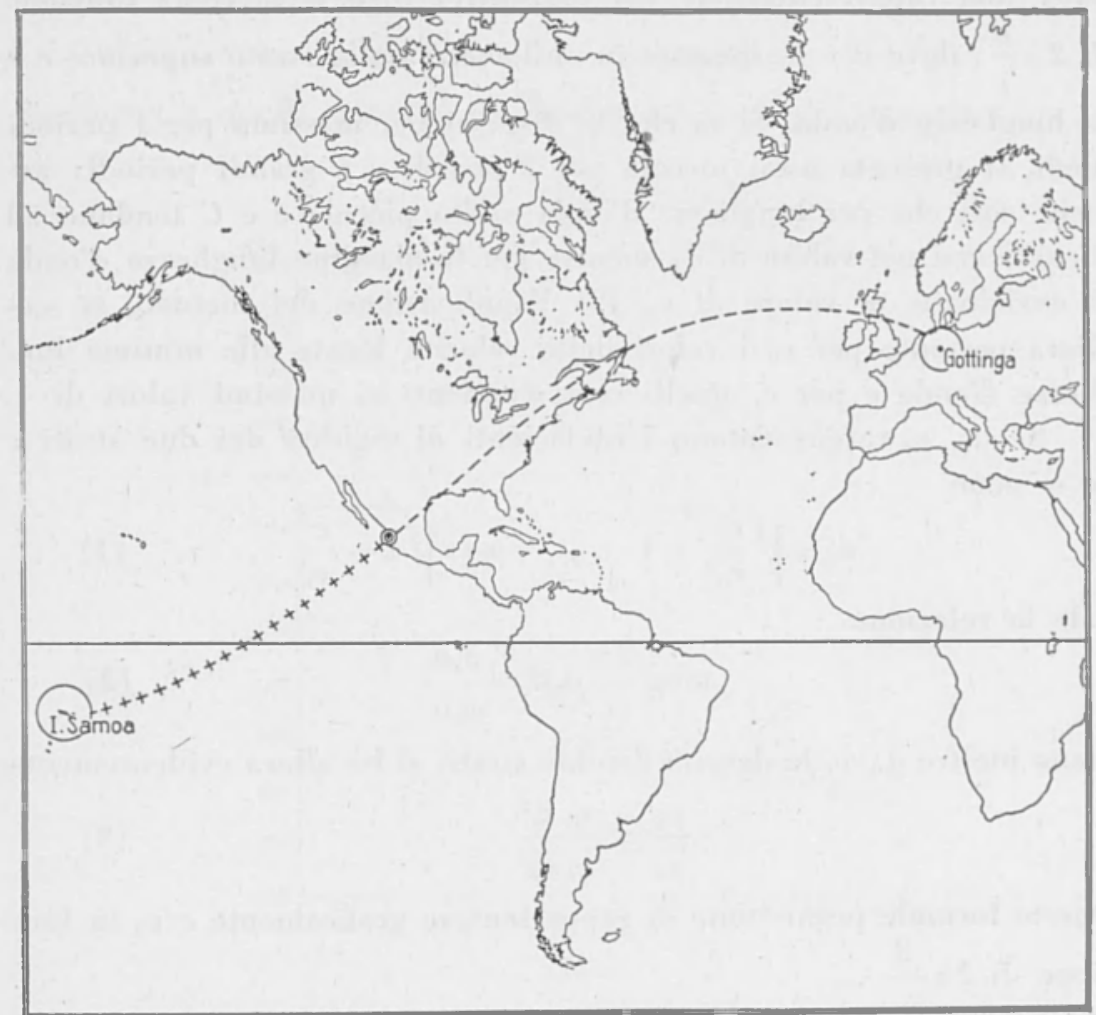

Fig. 2

chiaro che il tragitto Messico-Gottinga interessa l'Atlantico solo per una parte, e non la maggiore: dei $9700 \mathrm{~km}$ fra l'epicentro e Gottinga, solo $3100 \mathrm{~km}$ infatti riguardano l'Atlantico; il resto (più di 2/3) è tragitto continentale. Si spiega quindi perfettamente la diversità delle curve di dispersione osservata per i due tragitti.

2. Tentativo di determinazione dello spessore dello strato su cui poggia l'Oceano Atlantico. - Per questo tentativo, ci serviamo delle 
curve di dispersione delle onde di Love, registrate sulle rive limitanti l'Atlantico ad Est e ad Ovest, o nell'immediato retroterra.

Ricorreremo al primo lavoro di Jefíreys sull'argomento $\left({ }^{5}\right)$ che qui ci limiteremo a riassumere sommariamente e limitatamente ai fini che ci siamo proposti.

Indichiamo con $c_{0}$ e $c_{i}$ le velocità delle onde trasversali nello strato superiore e in quello che gli segue. Se $c$ e $C$ rappresentano le velocità di fase e di gruppo rispettivamente, la teoria di Jeffreys consente una rappresentazione dei rapporti $c / c_{0}$ e $C / c_{0}$ come funzione di $2 \pi \frac{d}{\lambda}$, dove $d$ è lo spessore in chilometri dello strato superiore e $\lambda$ la lunghezza d'onda. Si sa che la dispersione, massima per i periodi medi, si presenta assai piccola per i piccoli e i grandi periodi: avviene cosi che per lunghezze d'onda molto piccole $c$ e $C$ tendano ad identificarsi nel valore di $c_{0}$, mentre per le massime lunghezze d'onda si avvicinano al valore di $c_{i}$. Per l'applicazione del metodo, si sceglierà pertanto per $c_{0} \mathrm{i}$ valori delle velocità legate alle minime lunchezze d'onda e per $c_{i}$ quelli corrispondenti ai massimi valori di $\lambda$.

Se $\mu_{o}, \mu_{i}$ rappresentano $i$ coeflicienti di rigidità dei due strati e se si pone

$$
a_{\mathrm{o}}-\sqrt{\frac{c^{2}}{c_{\mathrm{o}}{ }^{2}}-1} \quad, \quad a_{\mathrm{i}}=\sqrt{1-\frac{c^{2}}{c_{\mathrm{i}}{ }^{2}}},
$$

vale la relazione

$$
\operatorname{tang} \frac{2 \pi}{\lambda} a_{\mathrm{o}} d-\frac{\mu_{\mathrm{i}} a_{\mathrm{i}}}{\mu_{\mathrm{o}} a_{\mathrm{o}}}
$$

Siano inoltre $\varrho_{o}, \varrho_{i}$ le densità dei due strati; si ha allora evidentemente

$$
\frac{\mu_{\mathrm{i}}}{\mu_{\mathbf{o}}}=\frac{\varrho_{\mathrm{i}} c_{\mathrm{i}}^{2}}{\varrho_{\mathbf{o}} c_{\mathbf{o}}^{2}} \text {. }
$$

Queste formule permettono di rappresentare graficamente $c / c_{0}$ in funzione di $2 \pi \frac{d}{i}$.

Si sa che la velocità di gruppo $C$ è legata alla velocità di fase $c$ mediante la formula

Consegue

$$
C=c-\lambda \frac{\partial c}{\partial \lambda}
$$

$$
\frac{C}{c_{\mathrm{o}}}=\frac{c}{c_{\mathrm{o}}}+2 \pi \frac{d}{\lambda} \frac{d\left(\frac{\boldsymbol{c}}{c_{\mathrm{o}}}\right)}{d\left(2 \pi \frac{d}{\lambda}\right)} .
$$


Mediante la [4] osservando che il quoziente differenziale nel secondo membro può essere dedotto dall'inclinazione della corrispondente curva per $c_{/}^{\prime} c_{0}$, si può calcolare $C / c_{0}$ come funzione di $2 \pi \frac{d}{\lambda}$.

Prima di passare all'applicazione, dobbiamo fare un'osservazione importante. In base ai risultati della ricerca precedente, noi riterremo pressoché trascurabile la presenza dello strato del "granito " ad est e ad ovest della soglia mediana atlantica. Ciò porta a scegliere per le densità $i$ seguenti valori:

$$
\varrho_{0}=3,0 \quad ; \quad \varrho_{i}=3,32 \text {, }
$$

conformi a quelli calcolati da Bullen per lo strato seguente il " gra-

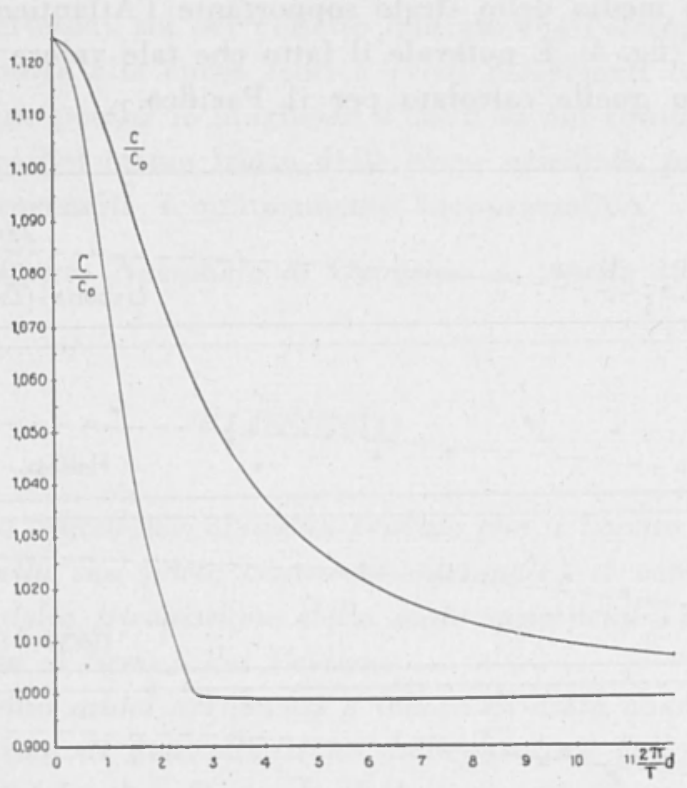

Fig. 3

nito » e il substrato ultrabasico. Le curve di dispersione relative alle onde $L_{a}$, quali furono registrate nelle sei stazioni scelte, ci hanno condotto ad assegnare alle velocità $\mathrm{i}$ seguenti valori

$$
c_{\mathrm{o}}=4,0 \quad, \quad c_{\mathrm{i}}=4,5 \mathrm{~km} / \mathrm{sec}
$$

Facendo uso delle [1], [2], [3] e [4] siamo pervenuti ai risultati rappresentati nella fig. 3

Per il calcolo dello spessore $d$ dello strato, le curve di dispersione danno il valore $c$ corrispondente ad ogni lunghezza d'onda. Noto $c$ 
dalla curva per $C_{/} c_{0}$ si trae il corrispondente valore per $2 \pi \frac{d}{\Lambda}$ e quindi lo spessore dello strato $d$.

Le grandezze in giuoco - particolarmente le lunghezze d'onda non possono essere dedotte che approssimativamente: perciò una valutazione per punti non può dare che risultati discordanti (salvo rare eccezioni, quali, nel nostro caso, Harvard, Ottawa, Lishona e Kew). I risultati possono essere così riassunti:

$\begin{array}{lrrrrrrrrr}\lambda \mathrm{km} . & 50 & 60 & 70 & 80 & 90 & 100 & 110 & 120 & 170 \\ d \mathrm{~km} & 18 & 19 & 18 & 14 & 15 & 15 & 17 & 18 & 17\end{array}$

Il valore medio dello strato sopportante l'Atlantico è quindi di $17 \mathrm{~km}$ circa (fig. 4). E notevole il fatto che tale valore coincide praticamente con quello calcolato per il Pacifico.

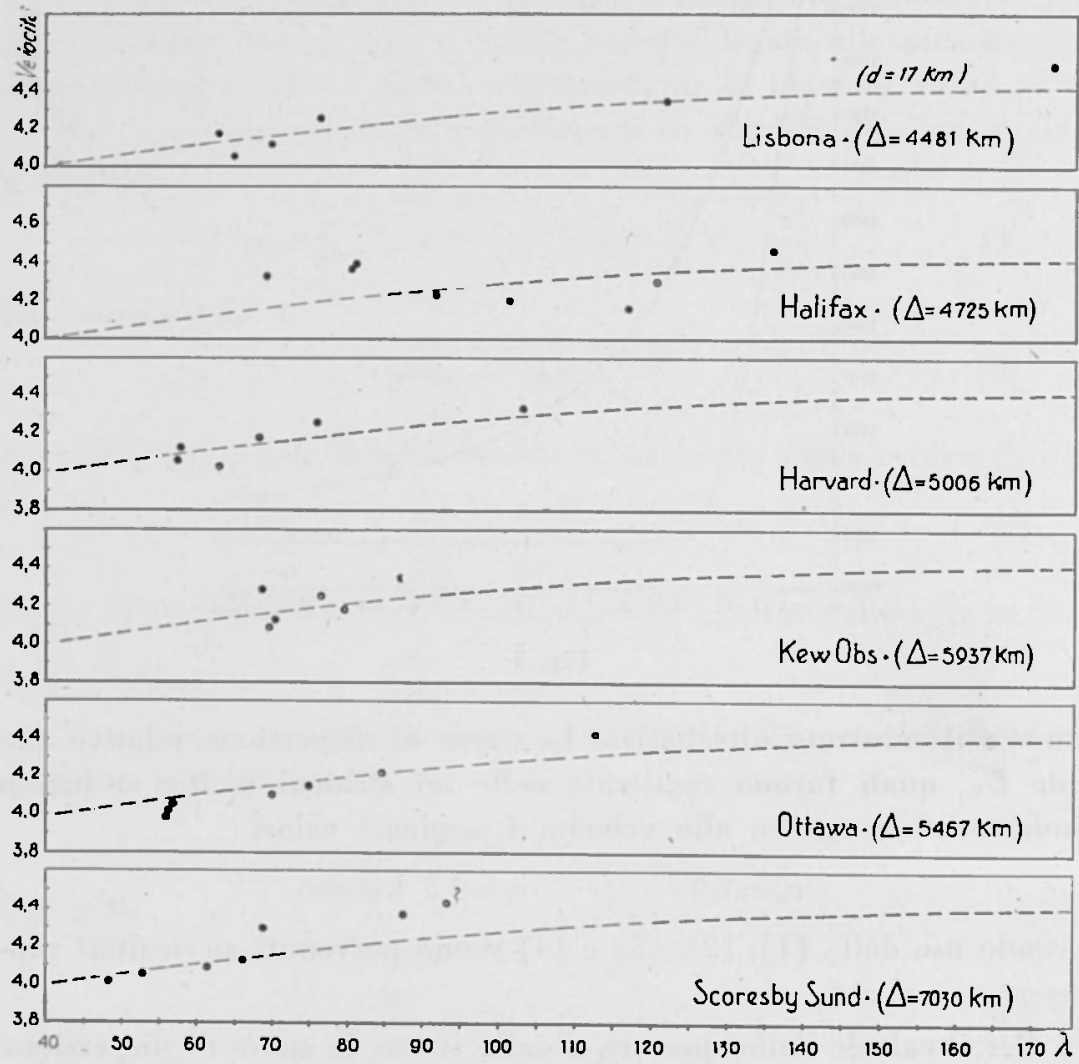

Fig. 4 
Come si è detto, ci siamo limitati alla teoria sviluppata da Jeffreys nel caso di due mezzi omogenei. Successivamente, Jeffreys ${ }^{\left({ }^{6-7}\right)}$ ampliò la sua ricerca, estendendola al caso di un mezzo (il secondo) eterogeneo. In questo caso, Jeffreys ottiene per la [2] l'espressione

$$
\operatorname{tang} \frac{2 \pi}{\lambda} a_{\mathrm{o}} d=\frac{\mu_{\mathrm{i}}}{\mu_{\mathrm{o}}} \frac{a_{\mathrm{i}}}{a_{\mathrm{o}}}\left\{1+\frac{1}{2} \frac{1+a_{\mathrm{i}}{ }^{2}}{L \frac{2 \pi}{\lambda} a_{\mathrm{i}}{ }^{3}}\left(1+\frac{1}{4 L \frac{2 \pi}{\lambda} a_{\mathrm{i}}}\right)\right\},
$$

in cui $L$ è una lunghezza costante. Quando $L=\infty$ (strato omogeneo) si ricade nella [2].

Abbiamo ritenuto superfluo ricorrere alla [2'] sia per la cennata difficoltà nella determinazione precisa della lunghezza d'onda (primaria causa di incertezza), sia per l'effetto limitato che l'eterogeneità del secondo strato porta alla curva teorica (vedi diagrammi calcolati da Jeffreys), sia infine perché le lunghezze d'onda da noi considerate portano ad usufruire del primo tratto delle curve calcolate, per il quale l'efletto dell'eterogeneità è praticamente inapprezzabile.

Roma - Istituto Nazionale di Geofisica - Aprile 1950.

\section{RIASSUNTO}

In un lavoro precedente abbiamo provato che il bacino dell' Atlantico (almeno nella sua parte centro-settentrionale) si comporta, dal punto di vista della trasmissione delle onde superficiali, in maniera del tutto analoga al bacino del Pacifico.

Qui si portano nuovi argomenti a favore di detta conclusione. Si fa poi un tentativo di determinazione dello spessore dello strato superficiale sotto l'Atlantico. Partendo dalle curve di dispersione delle onde $L_{4}$, e prescindendo dallo strato del a granito » ritenuto mancante o di entità trascurabile, si trova uno spessore del sottostante strato pari a $17 \mathrm{~km}$ ca., dell'ordine quindi di quello sopportante il Pacifico.

\section{BIBLIOGRAFIA}

(1) Caloi $P_{\rightarrow}$ Marcelli L., Pannocchia G.: Sulla velocità di propagazione delle onde superficiali in corrispondenza dell'Atlantico. Annali di Geofisica, II, 3 (1949).

(2) Leet L. D.: The Provincetown. Massachusetts, earthquake of April 23, 1935, 
and data for investigating New England's seismicity. Proceedings of the National Academy of Sciences, XXI, 6 (1935).

Hodcson J. H.: Analysis of travel times from Rockbursts at Kirkland Lake, Ontario. Bull. Seism. Soc. Amer., XXXVII, 1 (1947).

(3) Wilson James T. and Baykal Orhan: Crustal Structure of the North Atlantic Basin as Determined from Rayleigh-Wave Dispersion. Bull. Seism. Soc. Amer., XXXVIII, 1 (1948).

(4) Rohrbach Walter: Uber die Dispersion seismicher Oberflächenwellen. Zeitsch. f. Geophys, VIII, 3, 4 (1932).

(5) Jeffreys Harold: On the Surface Waves of Earthquakes. Monthl. Notic. Roy. Astr. Soc. Geophys. Suppl, I, 6 (1925).

(6) JefFreys HAROLd: The Effect on Love Waves of Heterogeneity in the Lou'er Layer. Monthl. Not. Roy. Astr. Soc. Geophys. Supplem., II, 2 (1928).

(7) Jeffreys Harold: The Surface Waves of Earthquakes. Mon. Not. Roy. Astr. Soc., Geophys. Suppl., III, 7 (1935). 